\title{
Towards personalizing treatment strategies in MCRPC: can dual-tracer PET-CT provide insights into tumor biology, guide the optimal treatment sequence, and individualize decision-making (between chemotherapy, second-generation anti-androgens and PSMA-directed radioligand therapy) early in the disease course?
}

\author{
Sandip Basu ${ }^{1,2} \cdot$ Rahul V. Parghane ${ }^{1,2} \cdot$ Sonam Suman ${ }^{1,2} \cdot$ Amit Joshi $^{2,3} \cdot K_{\text {Kumar Prabhash }}^{2,3} \cdot$ Ganesh Bakshi $^{2,4}$. \\ Sharmila Banerjee ${ }^{1,2}$
}

Published online: 27 November 2019

(C) Springer-Verlag GmbH Germany, part of Springer Nature 2019

\section{Introduction}

The therapeutic armamentarium of mCRPC has witnessed rapid evolution over the recent years, with a gamut of newly approved and "on-trial under clinical evaluation" therapies added to the traditional therapies including the cytotoxic chemotherapy (taxanes) and immunotherapy (Sipuleucel-T) [1]. These include (a) hormonal therapies such as secondgeneration anti-androgens namely, CYP17A1 inhibitors and androgen receptor (AR) antagonists; (b) systemically administered radiopharmaceuticals, e.g. PSMA-directed and bonedirected radionuclide therapies including alpha emitters; (c) PD-1 inhibitors and PARP inhibitors; and (d) bone-directed RANKL inhibitor denosumab. Amongst these, three powerful and potentially efficacious treatment options that hold great promise and being employed increasingly in mCRPC patients with large metastatic disease burden are (i) cytotoxic chemotherapy (e.g. docetaxel and cabazitaxel), (ii) newer antiandrogens (e.g. CYP17A1 inhibitor abiraterone acetate and

This article is part of the Topical Collection on Editorial

Sandip Basu

drsanb@yahoo.com

1 Radiation Medicine Centre, Bhabha Atomic Research Centre, Tata Memorial Hospital Annexe, Jerbai Wadia Road, Parel, Mumbai 400 012 , India

2 Homi Bhabha National Institute, Mumbai, India

3 Department of Medical Oncology, Tata Memorial Hospital, Mumbai, India

4 Department of Surgical Oncology, Tata Memorial Hospital, Mumbai, India
AR antagonist enzalutamide), and (iii) PSMA-directed systemic peptide receptor radioligand therapy (PRLT) with $\left[{ }^{177} \mathrm{Lu}\right] \mathrm{Lu}-\mathrm{PSMA} /\left[{ }^{225} \mathrm{Ac}\right] \mathrm{Ac}-\mathrm{PSMA}$. The concept of combining second-generation anti-androgen (abirateroneprednisolone) with $\left[{ }^{177} \mathrm{Lu}\right] \mathrm{Lu}-\mathrm{PSMA}$ PRLT for better and durable response is also frequently adopted in the practice and has been recently underscored in the literature [2], though the scientific data on the combination therapies is yet to evolve in this domain.

With multiple therapies in the horizon in MCRPC management, a need of the hour is to evolve rational optimal treatment sequencing and evolve a "step-care" management algorithm that could be employed in an individualized manner and will be developed on a scientific basis based upon tumour biology. Though there has been a mention on treatment sequencing in the management guidelines [1], a well-agreed upon consensus continues to be in its infancy.

\section{Cytotoxic chemotherapy and second-generation anti-androgens: the current role and place in $\mathrm{MCRPC}$ management}

Taxane-based docetaxel is considered to be one of the cornerstone first-line therapies in the management of mCRPC, the primary indications being patients who are either/or (i) symptomatic at presentation, (ii) presenting with visceral metastases, or (iii) asymptomatic disease but with a rapid PSA doubling time. A 3-weekly regimen of docetaxel in mCRPC documented improved overall survival (OS) and response in terms of pain reduction, PSA levels, and quality of life [3-5]. This has been proven by the landmark TAX327 study 
in 2004 and subsequently its updated analysis in 2008 [3-5]. Based upon the encouraging results obtained, the NICE advocated docetaxel as standard-of-care in $\mathrm{mCRPC}$ and first-line for symptomatic patients [6]. Resistance to docetaxel is primarily associated with increased expression of MDR1 gene that encodes P-glycoprotein, where cabazitaxel (7, 10dimethyloxy derivative of docetaxel) is proposed to be the next line of therapy (examined in the phase III TROPIC trial, an investigation conducted in 26 countries), and effective in docetaxel-resistant tumors because of its poor affinity for Pgp [7].

The new-generation hormonal therapies that have demonstrated considerable promise in $\mathrm{mCRPC}$ setting are abiraterone acetate and enzalutamide. The former has been proven to be effective in multiple prospective clinical trials and is now considered both as first and $2 \mathrm{nd} / 3 \mathrm{rd}$ line following resistance to chemotherapies [8-14]. The AR antagonist enzalutamide, on the other hand, received FDA and EMA approval based upon encouraging results obtained in the mCRPC pre-treated with docetaxel $[15,16]$. A recent phase III study (PREVAIL), conducted in more than 1700 patients with asymptomatic or minimally symptomatic progressive metastatic disease, has demonstrated considerable promise in chemotherapy-naive mCRPC patients with closure of the study in view of the statistically significant results obtained in the pre-planned interim analysis [17]. Based upon the results obtained, the new-generation hormonal therapies have been suggested as promising therapeutic agents in mCRPC both as first-line and in chemo-resistant scenario.

If one critically analyses the results obtained with the newgeneration anti-androgens, their efficacy in both chemotherapy-naïve and docetaxel-pre-treated patients would indicate that at least in a fraction of mCRPC patients, these would be the preferred choice as first-line and could be considered early in the disease course. Hence, one challenge lies in determining the subgroup of patients where this form of therapy could be considered upfront before administration of chemotherapy.

\section{PSMA-directed PRLT: can this be considered early in disease course?}

PSMA-targeted PRLT using small molecule PSMA inhibitors labelled with ${ }^{177} \mathrm{Lu} /{ }^{225} \mathrm{Ac}$ is a fast-evolving novel therapeutic option in patients of metastatic castration-resistant prostate cancer. The proof-of-the concept is evidenced by the multiple successful reports on $\left[{ }^{68} \mathrm{Ga}\right] \mathrm{Ga}-/\left[{ }^{177} \mathrm{Lu}\right] \mathrm{Lu}$-PSMA-based theranostics in mCRPC patients $[18-21]$. $\left[{ }^{68} \mathrm{Ga}\right] \mathrm{Ga}$-PSMA PET-CT has evolved as a sensitive molecular imaging technique to detect metastases in biochemically recurrent prostate cancer, even at low PSA levels and high uptake on $\left[{ }^{68} \mathrm{Ga}\right] \mathrm{Ga}$ PSMA scan is a pre-requisite for considering $\left[{ }^{177} \mathrm{Lu}\right] \mathrm{Lu}-$ PSMA PRLT. To date, however, $\left[{ }^{177} \mathrm{Lu}\right] \mathrm{Lu}$-PSMA has been and being clinically employed following failure of traditionally accepted chemotherapies and the newer anti-androgens, which makes this potentially efficacious and well-tolerated treatment option relatively underutilized.

We have to mention here that $\left[{ }^{177} \mathrm{Lu}\right]$ Lu-PSMA therapy, and also $\left[{ }^{68} \mathrm{Ga}\right] \mathrm{Ga} /\left[{ }^{18} \mathrm{~F}\right] \mathrm{F}$-PSMA PET-CT imaging, though being now frequently adopted in advanced centres with such facilities, are still considered experimental in many of the European countries, the USA and other countries worldwide. This scenario is however fast-changing and PSMA-based diagnostic and therapeutic approaches are likely to be extensively adopted in the near future, with the growth of prostate cancer theranostics in the coming years.

\section{Dual-tracer PET-CT in characterizing tumour biology of MCRPC: can this be employed for treatment individualization?}

The concept of dual/multi-tracer PET-CT in studying and characterizing tumor biology can be considered an important development of recent years that has scientifically augmented the practice of precision oncology. This concept has seen a major success in the clinical practice of neuroendocrine tumors (NET), where the combined somatostatin receptorbased PET and $\left[{ }^{18}\right.$ F]FDG PET is now routinely adopted to make personalized decision-making in addition to the Ki-67/ MIB-1 labelling index of the tumor [22, 23]. Over the years, we have observed that the attending physicians (including medical oncologists and nuclear medicine physicians) have given increasing importance to the dual-tracer PET imaging features in metastatic NET for deciding or choosing the appropriate strategy (PRRT versus chemotherapy versus combined chemo-PRRT).

Applying the similar analogy in MCRPC, it could be potentially possible to stratify the patients and individualize the available treatment strategies early in disease course. In our preliminary experience in $\mathrm{MCRPC}$ patients in $\left[{ }^{177} \mathrm{Lu}\right] \mathrm{Lu}-\mathrm{PSMA}$ PRLT setting, we have observed an evident association of high $\left[{ }^{18} \mathrm{~F}\right] \mathrm{FDG}$ uptake with increasing Gleason score and aggressive disease biology and are unlikely to respond favourably with PRLT alone, while those with $\left[{ }^{18} \mathrm{~F}\right] \mathrm{FDG}$-negative disease at times demonstrate excellent response with 2-3 cycles of PRLT despite having widespread disease at baseline (Fig. 1). In a recently published study [24] that critically examined in the setting of $\left[{ }^{177} \mathrm{Lu}\right] \mathrm{Lu}$-PSMA PRLT the value of $\left[{ }^{18} \mathrm{~F}\right] \mathrm{FDG}$ uptake in an unselected subset of highly pretreated patients of $\mathrm{mCRPC}$, there was an evident association of high $\left[{ }^{18} \mathrm{~F}\right] \mathrm{FDG}$ uptake with increasing Gleason score and poorer 12-month progression-free survival (PFS), indicative of aggressive disease biology. When these patients were sub-divided into groups $(\mathrm{Gp})$ as per 
Fig. 1 Excellent response to $\left.{ }^{177} \mathrm{Lu}\right]$-PSMA-617 PRLT

obtained in a 64-year-old male of mCRPC (Gleason score of 8; pretreated with bilateral orchidectomy and 9 cycles of docetaxel). Pre-therapy $\left[{ }^{68} \mathrm{Ga}\right] \mathrm{Ga}$-PSMA-11 PET-CT (a) showed PSMA expressing wide spread skeletal lesions (SUVmax-275) and pretherapy $\left[{ }^{18}\right.$ F]FDG PET-CT scan (b) shows no abnormal $\left[{ }^{18} \mathrm{~F}\right] \mathrm{FDG}$ uptake in skeletal lesions. The patient received 3 cycles of $\left.{ }^{177} \mathrm{Lu}\right] \mathrm{Lu}-\mathrm{PSMA}-617$ with posttherapy scan (c) showed good concentration of tracer in skeletal lesions. Follow-up $\left[{ }^{68} \mathrm{Ga}\right] \mathrm{Ga}$ PSMA-11 PET-CT (d) showed complete disappearance of abnormal PSMA tracer uptake in skeletal lesions with fall in serum PSA to $0.39 \mathrm{ng} / \mathrm{ml}$ after $2 \mathrm{nd}$ cycle of therapy and symptom-free status following therapy

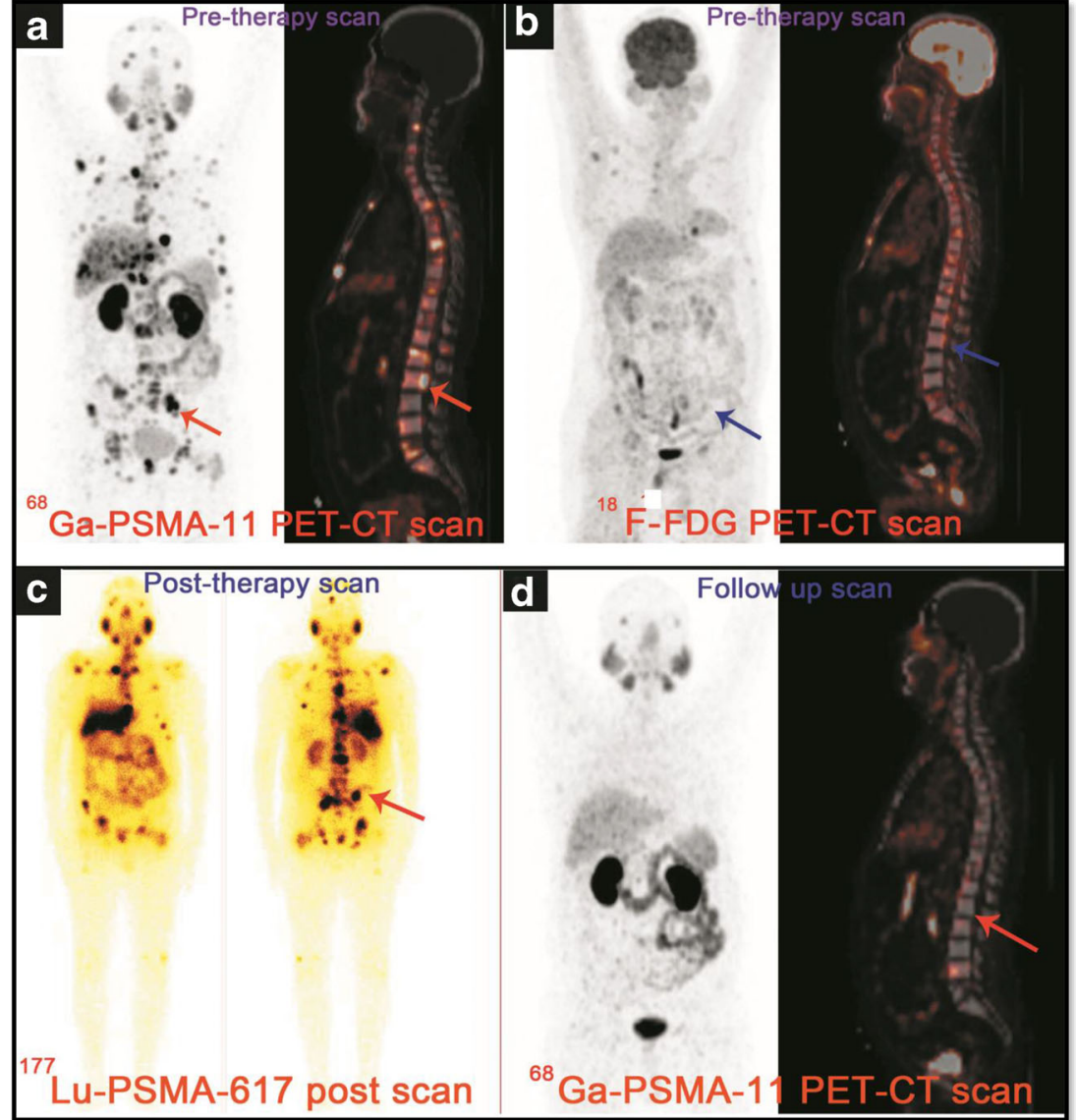

the $\left[{ }^{18} \mathrm{~F}\right] \mathrm{FDG}$ uptake (SUVmax) in the lesions (SUVmax values $0-7,7.1-15$ and $\geq 15.1$ ), with approximately equal distribution of cases in each, patients with Gleason score 8 and above showed higher $\left[{ }^{18} \mathrm{~F}\right]$ FDG uptake (SUVmax > 7.1). In the analysis for examining $\left[{ }^{18} \mathrm{~F}\right] \mathrm{FDG}$ uptake with clinical outcome following $\left[{ }^{177} \mathrm{Lu}\right] \mathrm{Lu}-\mathrm{PSMA}$ PRLT, $80 \%$ of the patients with SUVmax more than 15.1 showed progressive disease (PD), while the same outcome (i.e. PD) was observed in only half the magnitude $(40 \%)$ in the patients with SUVmax less than 15.

From theoretical perspectives, it is imperative that $\left[{ }^{18} \mathrm{~F}\right] \mathrm{FDG}$ avid disease with high Gleason score are likely to represent aggressive and undifferentiated disease that would be better responsive to taxane-based chemotherapy and could be potential candidates for this form of therapy, while those with $\left[{ }^{68} \mathrm{Ga}\right] \mathrm{Ga}$-PSMA-11 avid but $\left[{ }^{18} \mathrm{~F}\right]$ FDG-negative disease are likely to be responsive to $\left[{ }^{177} \mathrm{Lu}\right] \mathrm{Lu}-\mathrm{PSMA}-617$ PRLT and possibly, second-generation anti-androgens, making the latter subgroup suitable for these therapies, which could be considered early in the course of disease (Fig. 2). As mentioned before, the combination of $\left[{ }^{177} \mathrm{Lu}\right] \mathrm{Lu}-\mathrm{PSMA}$ and abiraterone with steroid can be conveniently employed in clinics (since, by theory, PSMA expression goes up in the androgen deprivation setting) [2]. Thus, dual-tracer PET-CT $\left(\left[{ }^{68} \mathrm{Ga}\right] \mathrm{Ga}-\right.$ PSMA-11 and $\left.\left[{ }^{18} \mathrm{~F}\right] \mathrm{FDG}\right)$ has the potential to be utilized as gatekeeper to rationally guide and individualize therapeutic strategies in patients of mCRPC (Fig. 3).

We have to mention here that a fraction of patients who initially are $\left[{ }^{18} \mathrm{~F}\right]$ FDG-negative may in their course of disease can become $\left[{ }^{18} \mathrm{~F}\right] \mathrm{FDG}$-positive indicating towards aggressive disease transformation. This underscores the value of dynamic disease prognostication with dualtracer PET-CT, which the nuclear medicine physicians experienced in theranostics are well familiar with. Such aggressive transformation is usually considered in the context of clinical/biochemical failures.

In summary, two noteworthy developments in the parlance of molecular imaging and radionuclide therapy that can be considered to have greatly augmented the practice of precision oncology are (i) development of multiple targeted therapeutic agents and (ii) ability to explore disease biology by dual/multi-tracer PET-CT. We believe both these developments may aid management rationalization and personalization in mCRPC setting through selection of appropriate therapies early in the course. Such endeavour would also possibly augment employing the promising and less toxic second-generation anti-androgens and $\left[{ }^{177} \mathrm{Lu}\right] \mathrm{Lu}$-PSMA PRLT early in the disease course and possibly could impact clinical outcome in this challenging group of patients. 


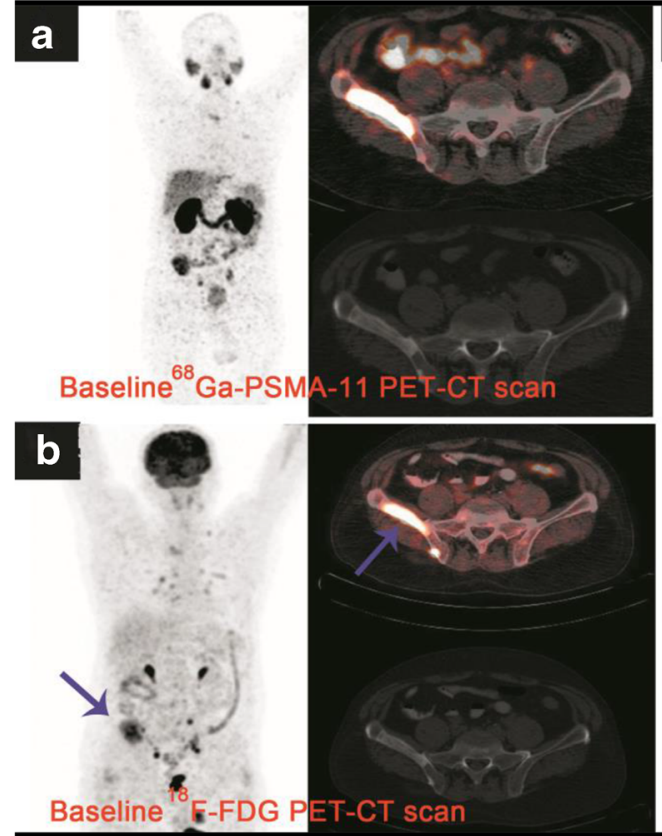

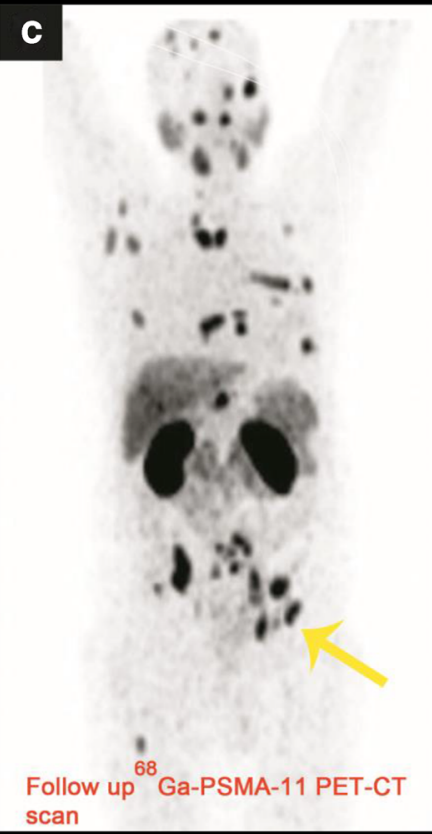

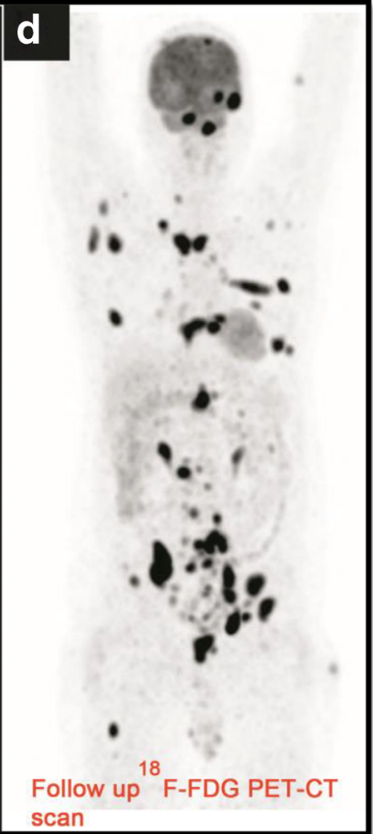

Fig. 2 Disease progression on PRLT in a diagnosed patient of conventional prostatic adenocarcinoma with $\left[{ }^{18} \mathrm{~F}\right] \mathrm{FDG}$ avid disease (Gleason score $5+4=9$; had earlier received leuprolide before undergoing bilateral orchidectomy; subsequently, 6 cycles of docetaxel and abiraterone followed by 3 cycles of cabazitaxel before considering for PRLT). Baseline pre-PRLT $\left[{ }^{68} \mathrm{Ga}\right] \mathrm{Ga}-\mathrm{PSMA}-11$ PET-CT (a) showed tracer avid iliac bone lesion with pelvic lymph nodes. Baseline $\left[{ }^{18}\right.$ F]FDG PET-CT (b) showed intense FDG uptake in skeletal and lymph nodal lesions. The patient received $\left[{ }^{177} \mathrm{Lu}\right] \mathrm{Lu}$-PSMA therapy, however in the follow-up $\left[{ }^{68} \mathrm{Ga}\right] \mathrm{Ga}$-PSMA and $\left[{ }^{18} \mathrm{~F}\right]$ FDG PET-CT scans showed appearance new lesions on both
Fig. 3 Proposed algorithm for rationalization and individualization of treatment strategies in MCRPC through dual-tracer PET-CT

\section{Metastatic Castration Resistant Prostate Cancer (already undergone medical/surgical castration)}

Progressive Disease

1. Dual tracer PET-CT based Molecular Imaging: ${ }^{68} \mathrm{Ga}-$ PSMA and ${ }^{18} \mathrm{~F}$ - FDG PET-CT

2. Gleason score
1. PSMA +ve
2. FDG +ve (high grade FDG uptake)
3. High Gleason score

Taxane-based Chemotherapy

(Docetaxel/Cabazitaxel)
Progressive disease

(Or Development of FDG positivity during follow-up)

\section{PSMA +ve}

2. FDG -ve (low grade FDG uptake)

3. Low Gleason score 
Acknowledgements The authors gratefully acknowledge the contribution of Dr B Basu in preparation of the schematic diagram.

\section{Compliance with ethical standards}

Conflict of interest The authors declare that they have no conflict of interest

Research involving human participants and/or animals Not applicable.

Informed consent Not applicable.

\section{References}

1. Scher HI, Halabi S, Tannock I, et al. Design and end points of clinical trials for patients with progressive prostate cancer and castrate levels of testosterone: recommendations of the Prostate Cancer Clinical Trials Working Group. J Clin Oncol. 2008;26:1148-59.

2. Basu S, Parghane RV, Joshi A, Prabhash K, Banerjee S. The rationality of combining second-generation antiandrogens with $177 \mathrm{Lu}-$ PSMA or its alpha-emitting congeners for better and durable results: will this dominate the therapeutic landscape and be an upfront consideration in metastatic castration-resistant prostate cancer in the coming years? Nucl Med Commun. 2018 Dec;39(12):1061-3.

3. Tannock IF, de Wit R, Berry WR, et al. Docetaxel plus prednisone or mitoxantrone plus prednisone for advanced prostate cancer. $\mathrm{N}$ Engl J Med. 2004;351:1502-12.

4. Petrylak DP, Tangen CM, Hussain MH, et al. Docetaxel and estramustine compared with mitoxantrone and prednisone for advanced refractory prostate cancer. N Engl J Med. 2004;351:1513-20.

5. Berthold DR, Pond GR, Soban F, de Wit R, Eisenberger M, Tannock IF. Docetaxel plus prednisone or mitoxantrone plus prednisone for advanced prostate cancer: updated survival in the TAX 327 study. J Clin Oncol. 2008:26:242-5.

6. National Institute for Health and Clinical Excellence. Docetaxel for the treatment of hormone-refractory metastatic prostate cancer. London: NICE; 2009.

7. de Bono JS, Oudard S, Ozguroglu M, et al. TROPIC Investigators. Prednisone plus cabazitaxel or mitoxantrone for metastatic castration-resistant prostate cancer progressing after docetaxel treatment: a randomised open-label trial. Lancet. 2010;376(9747): $1147-54$

8. Attard G, Reid AH, A'hern R, et al. Selective inhibition of CYP17 with abiraterone acetate is highly active in the treatment of castration-resistant prostate cancer. J Clin Oncol. 2009;27:3742-8.

9. Reid AH, Attard G, Danila DC, et al. Significant and sustained antitumor activity in post-docetaxel, castration-resistant prostate cancer with the CYP17 inhibitor abiraterone acetate. J Clin Oncol. 2010;28(9):1489-95.

10. Danila DC, Morris MJ, de Bono JS, et al. Phase II multicenter study of abiraterone acetate plus prednisone therapy in patients with docetaxel-treated castration-resistant prostate cancer. J Clin Oncol. 2010;28(9):1496-501.

11. Fizazi K, Scher HI, Molina A, et al. Abiraterone acetate for treatment of metastatic castration-resistant prostate cancer: final overall survival analysis of the COU-AA-301 randomised, double-blind, placebo-controlled Phase 3 study. Lancet Oncol. 2012;13:983-92.
12. Ryan CJ, Smith MR, de Bono JS, et al. Abiraterone in metastatic prostate cancer without previous chemotherapy. N Engl J Med. 2013;368:138-48.

13. Agarwal N, Hutson TE, Vogelzang NJ, et al. Abiraterone acetate: a promising drug for treatment of castration-resistant prostate cancer. Future Oncol. 2010;6(5):665-79.

14. Sonpavde G, Attard G, Bellmunt J, et al. The role of abiraterone acetate in the management of prostate cancer: a critical analysis of the literature. Eur Urol. 2011;60(2):270-8.

15. Scher HI, Beer TM, Higano CS, et al. Antitumour activity of MDV3100 in castration-resistant prostate cancer: a Phase 1-2 study. Lancet. 2010;375:1437-46.

16. Scher HI, Fizazi K, Saad F, et al. Increased survival with enzalutamide in prostate cancer after chemotherapy. N Engl J Med. 2012;367:1187-97.

17. Beer TM, Armstrong AJ, Sternberg $\mathrm{CN}$ et al. Enzalutamide in men with chemotherapy-naive metastatic prostate cancer (mCRPC): results of phase III PREVAIL study. Presented at: 2014 Genitourinary Cancer Symposium. San Francisco, CA, USA, 29 January-2 February 2014.

18. Baum RP, Kulkarni HR, Schuchardt C, Singh A, Wirtz M, Wiessalla S, et al. 177Lu-Labeled prostate-specific membrane antigen radioligand therapy of metastatic castration-resistant prostate cancer: safety and efficacy. J Nucl Med. 2016;57(7):1006-13.

19. Hofman MS, Violet J, Hicks RJ, Ferdinandus J, Thang SP, Akhurst $\mathrm{T}$, et al. [ $\left.{ }_{177} \mathrm{Lu}\right]-\mathrm{PSMA}-617$ radionuclide treatment in patients with metastatic castration-resistant prostate cancer (LuPSMA trial): a single-centre, single-arm, phase 2 study. Lancet Oncol. 2018;19(6):825-33.

20. Kulkarni HR, Singh A, Langbein T, Schuchardt C, Mueller D, Zhang $\mathrm{J}$, et al. Theranostics of prostate cancer: from molecular imaging to precision molecular radiotherapy targeting the prostate specific membrane antigen. Br J Radiol. 2018;1:20180308. https:// doi.org/10.1259/bjr.20180308.

21. Yadav MP, Ballal S, Tripathi M, Damle NA, Sahoo RK, Seth A, et al. ${ }^{177}$ Lu-DKFZ-PSMA-617 therapy in metastatic castration resistant prostate cancer: safety, efficacy, and quality of life assessment. Eur J Nucl Med Mol Imaging. 2017;44(1):81-91.

22. Basu S, Sirohi B, Shrikhande SV. Dual tracer imaging approach in assessing tumor biology and heterogeneity in neuroendocrine tumors: its correlation with tumor proliferation index and possible multifaceted implications for personalized clinical management decisions, with focus on PRRT. Eur J Nucl Med Mol Imaging. 2014;41(8):1492-6.

23. Basu S, Ranade R, Thapa P. Correlation and discordance of tumour proliferation index and molecular imaging characteristics and their implications for treatment decisions and outcome pertaining to peptide receptor radionuclide therapy in patients with advanced neuroendocrine tumour: developing a personalized model. Nucl Med Commun. 2015;36(8):766-74.

24. Suman S, Parghane RV, Joshi A, Prabhash K, Bakshi G, Talole S, et al. Therapeutic efficacy, prognostic variables and clinical outcome of (177)Lu-PSMA-617 PRLT in progressive mCRPC following multiple lines of treatment: prognostic implications of high FDG uptake on dual tracer PET-CT vis-à-vis Gleason Score in such cohort. Br J Radiol. 2019;10:20190380. https://doi.org/10.1259/bjr. 20190380.

Publisher's note Springer Nature remains neutral with regard to jurisdictional claims in published maps and institutional affiliations. 